\title{
Correction to: Is it possible to disregard obsolete requirements? A family of experiments in software effort estimation
}

\author{
Lucas Gren ${ }^{1,2,3}$ (D) Richard Berntsson Svensson ${ }^{3}$
}

Published online: 4 May 2021

(c) Springer-Verlag London Ltd., part of Springer Nature 2021

\section{Correction to: Requirements Engineering https://doi.org/10.1007/s00766-021-00351-7}

In the original publication of the article, the affiliation of author Lucas Gren has been incorrectly published.

The correct affiliation should read as below:

1: Blekinge Institute of Technology, Karlskrona, Sweden.

2: Volvo Cars, Gothenburg, Sweden.

3: Chalmers and The University of Gothenburg, Gothenburg, Sweden.

The original article has been corrected.
Publisher's Note Springer Nature remains neutral with regard to jurisdictional claims in published maps and institutional affiliations.

The original article can be found online at https://doi.org/10.1007/ s00766-021-00351-7.

Lucas Gren

lucas.gren@bth.se

Richard Berntsson Svensson

richard@cse.gu.se

1 Blekinge Institute of Technology, Karlskrona, Sweden

2 Volvo Cars, Gothenburg, Sweden

3 Chalmers and The University of Gothenburg, Gothenburg, Sweden 